\title{
Analysis of the relationship between serum amyloid protein A, procalcitonin, C-reactive protein, and peripherally inserted central catheter infection in patients with malignant tumor
}

\author{
Junyao Chen ${ }^{1}$, Jinjing Huang ${ }^{1}$, Tao Wang ${ }^{2}$, Chenggang Xie ${ }^{3}$ \\ ${ }^{1}$ Laboratory Medicine Department, Gansu Provincial Cancer Hospital, Lanzhou, China; ${ }^{2}$ Translational Medicine Research Center, Gansu Provincial \\ Cancer Hospital, Lanzhou, China; ${ }^{3}$ Laboratory Medicine Department, First People's Hospital of Tianshui, Tianshui, China \\ Contributions: (I) Conception and design: J Chen, C Xie; (II) Administrative support: C Xie; (III) Provision of study materials or patients: J Huang; (IV) \\ Collection and assembly of data: J Huang, T Wang; (V) Data analysis and interpretation: J Chen, C Xie; (VI) Manuscript writing: All authors; (VII) \\ Final approval of manuscript: All authors. \\ Correspondence to: Chenggang Xie. Department of Laboratory, Tianshui First People’s Hospital, Tianshui 741000, China. Email: xcg15@163.com.
}

Background: Compared with a central venous catheter, a peripherally inserted central catheter (PICC) has the advantages of less trauma and a lower risk of bleeding and bloodstream infection. However, patients with tumors are commonly immunocompromised and therefore susceptible to infection during the course of PICC indwelling. This study aimed to investigate the relationship between serum amyloid protein A (SAA), procalcitonin (PCT), C-reactive protein (CRP), and PICC infection in patients with malignant tumor.

Methods: Patients with malignant tumor treated with PICC in Gansu Provincial Cancer Hospital from March 2017 to January 2020 were retrospectively investigated. The incidence of infection and the differences in SAA, PCT, CRP, prothrombin time (PT), activated partial thromboplastin time (APTT), thrombin time (TT), fibrinogen (FIG), interleukin-18 (IL-18), and interleukin-16 (IL-6) between the infected group and noninfected group were observed. The correlations of SAA, PCT, and CRP levels in different infection sites and infection degrees were analyzed.

Results: A total of 414 eligible patients were included with 93 in the infection group and 321 in the noninfection group. The overall incidence of infection was $22.4 \%$. The levels of SAA, PCT and CRP in the infection group were significantly higher than those in the noninfection group $(\mathrm{P}<0.001)$. Compared with the non-infected group, the levels of PT, APTT, TT, IL-18 and IL-6 were significantly higher in the infected group $(\mathrm{P}<0.001)$. However, the level of FIG was significantly lower in the infected group $(\mathrm{P}<0.001)$. Compared with the local infection group, the levels of SAA, PCT, and CRP in the bloodstream infection group were significantly higher $(\mathrm{P}<0.001)$. Furthermore, the levels of SAA, PCT, and CRP were positively correlated with the degree of PICC catheter infection.

Conclusions: In patients with malignant tumors, PICC catheter infection were associated with increased risk of bleeding and the levels of SAA, PCT, and CRP can be used as predictive diagnostic indexes for PICC catheter infection. Furthermore, these parameters were positively correlated with the degree of PICC catheter infection.

Keywords: Serum amyloid protein A, procalcitonin; C-reactive protein, malignant tumor, peripherally inserted central catheter, infection

Submitted Jan 26, 2021. Accepted for publication May 17, 2021.

doi: 10.21037/apm-21-796

View this article at: http://dx.doi.org/10.21037/apm-21-796 


\section{Introduction}

Malignant tumors have the characteristics of aggressive invasiveness, metastasis, dysfunction caused by organ invasion, and high morbidity and mortality in clinic. At present, chemotherapy remains the main approach in clinical treatment for malignant tumors. However, chemotherapeutic drugs are irritants and are highly toxic, and, once these drugs enter the bloodstream, they can easily cause local tissue damage. Peripherally inserted central catheter (PICC) provides a safe and effective way of intravenous administration for patients with malignant tumors. Moreover, PICC catheterization has the advantages of simple and convenient operation, good safety, and a high success rate. Moreover, it can lessen the pain caused by repeated puncture and reduce the occurrence of phlebitis $(1,2)$. However, PICC catheterization is an invasive operation, and the long course of malignant tumor and the effects of chemotherapy and other factors may compromise the immunity of patients and increase the risk of infection (3). The averaged incidence of PICCrelated complications and infection in cancer patients was $33.2 \%$ and $12.6 \%$, respectively (4). In China, the incidence of PICC catheterization-related blood stream infection (BSI) was $1.3 \%$, which seriously affects the treatment compliance of malignant tumor patients (5). In addition, it is not conducive to the rehabilitation of patients, and even lead to death $(6,7)$. Serum concentrations of PCT and CRP increase greatly after the onset of bacterial infection in patients with cancer (8). As a consequence, early and effective laboratory detection of these biomarkers have important clinical value for early diagnosis and effective management of PICC catheter infection in patients with malignant tumors. The purpose of this study was thus to further explore the relationship between serum amyloid protein A (SAA), procalcitonin (PCT), C-reactive protein (CRP), and 4 items of coagulation and inflammatory factors with PICC catheter infection in patients with malignant tumor. We hypotheses that the PICC-related infections could be accurately diagnosed by detecting the serum levels of these biomarkers. We present the following article in accordance with the STROBE reporting checklist (available at http://dx.doi.org/10.21037/apm-21-796).

\section{Methods}

\section{Study design}

This is a retrospective observational study which was carried out in a tertiary hospital in Lanzhou China. This study was approved by the ethical committee of Gansu Provincial Cancer Hospital and was performed in accordance with the Helsinki Declaration (as revised in 2013). The informed consent was waived due to the retrospective nature and anonymous data.

\section{Participants}

Patients with malignant tumors who were treated with PICC from March 2017 to January 2020 in Gansu Provincial Cancer Hospital were retrospectively investigated. Patients inclusion criteria were as follow: (I) patients with malignant tumors who received chemotherapy; (II) patients without serious immune system disease; (III) patients with complete clinical data; (IV) patients who received PICC catheterization; and (V) patients with sound mind and good compliance. Meanwhile, the exclusion criteria were the following: (I) patients with infectious diseases; (II) patients with mental disorders; or (III) patients with incomplete clinical data.

\section{Study outcomes}

The incidence of infection was observed, and the levels of SAA, PCT, CRP, blood coagulation (PT, APTT, TT, and FIG), and inflammatory factors (IL-18, IL-6) were compared between the infected group and the noninfected group. Moreover, the levels of SAA, PCT, and CRP in different sites in the noninfected group and the noninfected group were analyzed.

\section{Specimen testing method and apparatus}

After patients had fasted for more than 8 hours, the elbow venous blood was collected in the morning and placed in sodium citrate anticoagulation tubes and ordinary dry coagulation-promoting tubes respectively. The specimens were centrifuged at $3,000 \mathrm{r} / \mathrm{min}$ for 15 minutes to separate serum and plasma. The plasma and serum were stored in the refrigerator at $-20^{\circ} \mathrm{C}$ after separation. The level of SAA was detected by scatter turbidimetry with a Sysmex CA-600 special protein analyzer (Kobe, Japan). PCT and CRP were detected by a Cobas 8000 automatic biochemical immune pipeline (Roche Diagnostics). Interleukin 18 (IL-18) and interleukin 16 (IL-6) were detected by double-antibody sandwich enzyme linked immunosorbent assay (ELISA) 
Table 1 Comparison of SAA, PCT, CRP and levels between the infection group and noninfection group $(\bar{x} \pm s)$

\begin{tabular}{lccc}
\hline Group & SAA $(\mathrm{mg} / \mathrm{L})$ & $\mathrm{PCT}(\mathrm{ng} / \mathrm{mL})$ & $\mathrm{CRP}(\mathrm{mg} / \mathrm{L})$ \\
\hline Infection group $(\mathrm{n}=93)$ & $284.484 \pm 149.783$ & $3.392 \pm 2.424$ & $80.798 \pm 68.713$ \\
Noninfection group $(\mathrm{n}=321)$ & $38.190 \pm 19.551$ & $0.345 \pm 0.017$ & $9.902 \pm 2.253$ \\
$\mathrm{t}$ & 15.818 & 13.358 & 9.948 \\
$\mathrm{P}$ & $<0.001$ & $<0.001$ & $<0.001$ \\
\hline
\end{tabular}

SAA, serum amyloid protein A; PCT, procalcitonin; CRP, C-reactive protein.

with a MINI VIDAS automated immunoanalyzer (bioMérieux, Marcy-l'Étoile, France). Prothrombin time (PT), activated partial thromboplastin time (APTT), thrombin time (TT), and fibrinogen (FIG) were detected by the Sysmex 5100 automatic coagulation analysis system. The above kits were purchased from the same company, and matching reagents were produced by the same company as its analyzer. In addition, the operation steps were carried out strictly according to the manufacturer's instructions.

\section{Statistical analysis}

All the statistical analyses were performed by using SPSS 22.0 statistical software (IBM, Corp., Armonk, NY, USA). The continuous variables with normal distribution were expressed as mean \pm standard deviation and were compared using the $t$-test. The levels of inflammatory indicators in each group were expressed by box plots. The differences among groups were further compared by Mann-Whitney test.

\section{Results}

\section{Patients inclusion}

A total of 414 eligible patients were included. The baseline characteristics were as follows: 261 males and 153 females, aged from 20 to 83 years (mean age 64.23 years), with an averaged indwelling time of 182.08 days. The patients included those with lung cancer $(\mathrm{n}=132)$, gastric cancer $(n=90)$, liver cancer $(n=71)$, esophageal cancer $(n=64)$, breast cancer $(n=35)$, and osteosarcoma $(n=22)$. The patients were divided into an infection group $(\mathrm{n}=93)$ and a noninfection group $(n=321)$ according to whether the malignant tumor patients were infected or not. There was no significant difference in baseline data between the 2 groups $(\mathrm{P}>0.05)$.

\section{Analysis of the incidence of PICC catheter infection in patients with malignant tumor}

Among the 414 cases of patients with malignant tumor and PICC catheterization, 93 cases were observed to have infection, yielding an infection rate of $22.46 \%$. Of these, there were 74 cases of local infection, with an incidence of $79.57 \%$, and 19 cases of bloodstream infection, with an incidence of $20.43 \%$, according to the site of infection.

\section{Comparative analysis of SAA, PCT, and CRP levels between the infection group and the noninfection group}

The levels of SAA, PCT, and CRP in the infection group were significantly higher than those in the non-infection group $(\mathrm{P}<0.001 ;$ Table 1$)$.

\section{Comparative analysis of blood coagulation function between the infection group and the noninfection group}

Compared with the noninfection group, the levels of PT, APTT, and TT were significantly higher in the infection group. Whereas, the level of FIG in the infected group were significantly lower than that in the non-infected group $(\mathrm{P}<0.001 ;$ Table 2).

\section{Comparative analysis of the level of inflammatory factors between the infection group and the noninfection group}

The levels of IL-18 and IL-6 in the infected group were significantly higher than those in the noninfected group $(\mathrm{P}<0.001 ;$ Table 3).

\section{Comparison of SAA, PCT, and CRP levels at different infection sites}

The levels of SAA, PCT, and CRP in the bloodstream 
Table 2 Comparison of PT, APTT, TT, and FIG levels between the infection group and noninfection group $(\bar{x} \pm s)$

\begin{tabular}{lcccc}
\hline Group & APTT $(\mathrm{s})$ & PT $(\mathrm{s})$ & TT $(\mathrm{s})$ & FIG $(\mathrm{g} / \mathrm{L})$ \\
\hline Infection group $(\mathrm{n}=93)$ & $38.33 \pm 2.79$ & $17.01 \pm 1.59$ & $20.11 \pm 2.03$ & $1.79 \pm 0.45$ \\
Noninfection group $(\mathrm{n}=321)$ & $30.81 \pm 1.64$ & $13.42 \pm 1.23$ & $15.07 \pm 1.42$ & $2.97 \pm 0.58$ \\
$\mathrm{t}$ & 24.782 & 20.101 & 22.407 & -20.777 \\
$\mathrm{P}$ & $<0.001$ & $<0.001$ & $<0.001$ & $<0.001$ \\
\hline
\end{tabular}

PT, prothrombin time; APTT, activated partial thromboplastin time; TT, thrombin time; FIG, fibrinogen.

Table 3 Comparison of IL-18 and IL-6 levels between the infection group and noninfection group $(\bar{x} \pm s)$

\begin{tabular}{lcc}
\hline Group & $\mathrm{IL}-18(\mathrm{mg} / \mathrm{L})$ & $\mathrm{IL}-6(\mathrm{mg} / \mathrm{L})$ \\
\hline Infection group $(\mathrm{n}=93)$ & $46.35 \pm 5.07$ & $12.47 \pm 2.87$ \\
Noninfection group $(\mathrm{n}=321)$ & $21.68 \pm 1.93$ & $6.95 \pm 1.37$ \\
$\mathrm{t}$ & 45.970 & 17.965 \\
$\mathrm{P}$ & $<0.001$ & $<0.001$ \\
\hline
\end{tabular}

IL-18, interleukin-18; IL-6, interleukin-16.

Table 4 Comparison of SAA, PCT, and CRP levels at different infection sites $(\bar{x} \pm s)$

\begin{tabular}{lccc}
\hline Group $(\mathrm{n})$ & SAA $(\mathrm{mg} / \mathrm{L})$ & PCT $(\mathrm{ng} / \mathrm{mL})$ & $\mathrm{CRP}(\mathrm{mg} / \mathrm{L})$ \\
\hline Local infection $(\mathrm{n}=74)$ & $218.180 \pm 41.430$ & $2.246 \pm 0.559$ & $57.460 \pm 54.448$ \\
Bloodstream infection $(\mathrm{n}=19)$ & $542.718 \pm 138.718$ & $7.879 \pm 1.511$ & $171.693 \pm 33.901$ \\
$\mathrm{t}$ & 10.083 & 15.923 & 11.392 \\
$\mathrm{P}$ & $<0.001$ & $<0.001$ & $<0.001$ \\
\hline
\end{tabular}

SAA, serum amyloid protein A; PCT, procalcitonin; CRP, C-reactive protein.

infection group were significantly higher than those in the local infection group $(\mathrm{P}<0.001 ;$ Table 4$)$.

\section{Analysis of the relationship between the levels of $S A A$, PCT, CRP, and the degree of PICC catheter infection in patients with malignant tumor}

It was found that there were significant differences in SAA, PCT, and CRP levels across the noninfection group, local infection group, and bloodstream infection group $(\mathrm{P}<0.001$; Figure 1).

\section{Discussion}

Our present study demonstrated that serum SAA, PCT, and CRP can be used as predictors for PICC-related infection in cancer patients treated with PICC. Furthermore, these parameters were positively correlated with the severity of PICC-related infection.

According to related studies $(3,6)$, the use of PICC catheterization in patients with malignant tumor can effectively improve the therapeutic effect. However, it is an invasive operation and is affected by various factors in the clinical practice. In addition, patients with malignant tumor need to retain the PICC catheter for an extended period. Additionally, administration of chemotherapeutic drugs causes further irritation and reduces the immune function of patients with malignant tumor, leaving them more prone to PICC catheter infection. As a consequence, the prevention and improvement of PICC catheter infection in patients with malignant tumors has become one of the important topics in clinical research.

The acute phase proteins including SAA, PCT, and CRP are positively associated with the severity of the acute 

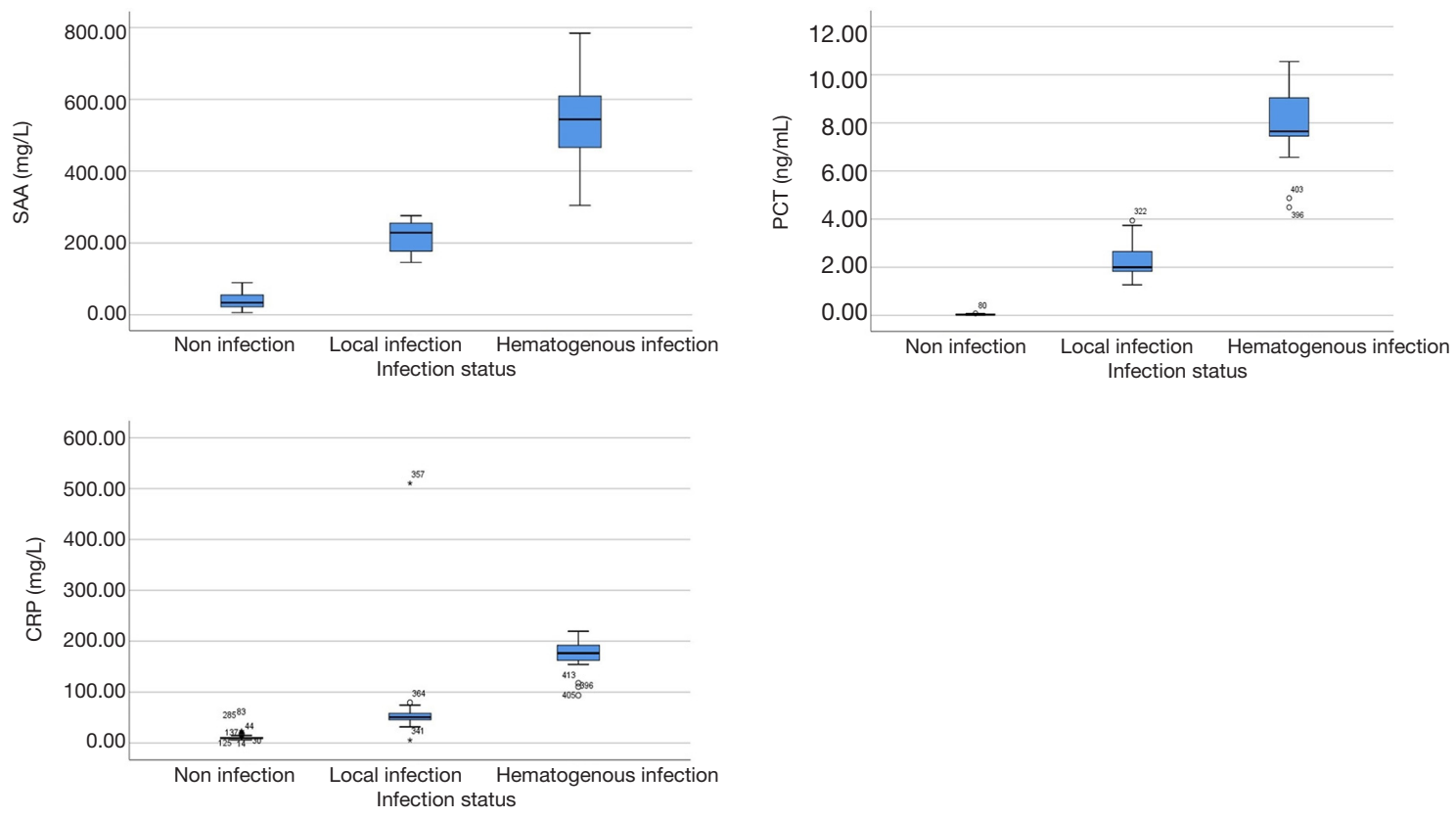

Figure 1 Comparison of SAA, PCT, and CRP levels among patients with different degrees of infection. SAA, serum amyloid protein A; PCT, procalcitonin; CRP, C-reactive protein.

inflammatory response. Their levels in serum increase when acute infection occurs (4). The level of SAA, a sensitive acute phase reactive protein, can increase rapidly within 4 6 hours and can reach more than 1,000 times the normal level when chronic or acute inflammation occurs (9). It has the characteristics of early rise, wide range, high sensitivity, and a rapid decrease to normal level after pathogen elimination. It is a sensitive predictor for infection and inflammation recovery. PCT is a protein containing 116 amino acids. PCT levels increase rapidly and have a long half-life in severe bacterial infection and sepsis. However, PCT does not increase in autoimmunity, allergy, and virus infection. As a consequence, the serum level of PCT can reflect the infection status in the early stage and was used to assess the severity of infection $(10,11)$. CRP is an acute protein in the blood that rises rapidly during infection or trauma and can be used as a nonspecific marker of systemic inflammation. The level of CRP in normal serum is very low and stable, but will increase with time and peak at 22-28 hours following infection or trauma (6). It can affect the immunomodulation and phagocytosis of invading cells. The conjugated complex can activate the complement system and finally be expressed through inflammatory response $(1,7)$.

The inflammatory mediators and cytokines in the blood can cross-react with the coagulation system after the inflammatory reaction occurs (12). Especially in the early stage of sepsis, the levels of a large number of inflammatory mediators, such as IL-18 and IL-6, increase. This can lead to diffuse vascular endothelial injury and expose the collagen under the vascular endothelium. It can also promote the release of coagulation factor XII and platelet-activating factor, leading to the activation of endogenous coagulation pathway and subsequent coagulation dysfunction (13). Our results showed that the levels of PT, APTT, TT, IL-18, and IL-6 in the infection group were significantly higher than those in the noninfection group $(\mathrm{P}<0.001)$. However, the level of FIG in infection group was significantly lower than that in the noninfection group $(\mathrm{P}<0.001)$. It is suggested that PICC catheter infection in patients with malignant tumor can promote the release of a large number of inflammatory factors, cause coagulation system disorder, prolong coagulation time, and increase the risk of bleeding.

Among the 414 patients with malignant tumors treated with PICC catheterization, 93 were observed to have infection, with an infection rate of $22.46 \%$. Among the patients with PICC infection, 74 were local infections, with an incidence of $79.57 \%$, and 19 cases were bloodstream infections, with an incidence of $20.43 \%$. The levels of SAA, PCT and CRP in the infection group were significantly higher than those in the noninfection group $(\mathrm{P}<0.001)$, 
which was in line with the results of Niu et al. (14). Additionally, the levels of SAA, PCT, and CRP in the bloodstream infection group were significantly higher than those in the local infection group $(\mathrm{P}<0.001)$. This suggests that the levels of SAA, PCT, and CRP may provide an important information in the differential diagnosis of PICC catheter infection in patients with malignant tumors. We also compared the differences of these infection indicators among noninfection, local infection, and bloodstream infection groups. Our findings showed that there were significant differences in the levels of SAA, PCT, and CRP across the three groups $(\mathrm{P}<0.001)$. Thus, these indices may serve as important parameters for assessing the severity of infection.

\section{Limitations}

There exist some limitations in our present study. First, the retrospective design may lead to data and information bias. Second, the possible causal relationship between PICCrelated infection and the derangement of aforementioned biomarkers was compromised by the retrospective nature of the present study. Last but not the least, this study was a single research and the findings could be not representative of PICC outcomes in other settings. Studies with multicenter, prospective design, and large sample size are warranted in this field.

In conclusion, the levels of SAA, PCT, and CRP can be used as predictors of PICC catheter infection in patients with malignant tumors. Meanwhile, the levels of SAA, PCT, and CRP may serve as important parameters for assessing the degree of PICC catheter infection in patients with malignant tumors.

\section{Acknowledgments}

Funding: None.

\section{Footnote}

Reporting Checklist: The authors have completed the STROBE reporting checklist. Available at http://dx.doi. org/10.21037/apm-21-796

Data Sharing Statement: Available at http://dx.doi. org/10.21037/apm-21-796

Conflicts of Interest: All authors have completed the ICMJE uniform disclosure form (available at http://dx.doi. org/10.21037/apm-21-796). The authors have no conflicts of interest to declare.

Ethical Statement: The authors are accountable for all aspects of the work in ensuring that questions related to the accuracy or integrity of any part of the work are appropriately investigated and resolved. This study was approved by the ethical committee of Gansu Provincial Cancer Hospital (No. P202103080003) and was performed in accordance with the Helsinki Declaration (as revised in 2013). The informed consent was waived due to the retrospective nature and anonymous data.

Open Access Statement: This is an Open Access article distributed in accordance with the Creative Commons Attribution-NonCommercial-NoDerivs 4.0 International License (CC BY-NC-ND 4.0), which permits the noncommercial replication and distribution of the article with the strict proviso that no changes or edits are made and the original work is properly cited (including links to both the formal publication through the relevant DOI and the license). See: https://creativecommons.org/licenses/by-nc-nd/4.0/.

\section{References}

1. Purran A, Weller G, Kerr C. Evaluation of a PICC care training programme. Nurs Stand 2016;30:45-50.

2. Kang J, Liu B, Sun W. Two successful insertions of peripherally inserted central catheters in a patient with persistent left superior vena cava: A case report. Medicine (Baltimore) 2019;98:e16988.

3. Huang ZP, Liu XJ, Zou BX, et al. The complete recanalization of PICC-related venous thrombosis in cancer patients: A series of case reports. Exp Ther Med 2013;6:411-2.

4. Seckold T, Walker S, Dwyer T. A comparison of silicone and polyurethane PICC lines and postinsertion complication rates: a systematic review. J Vasc Access 2015;16:167-77.

5. Kang J, Chen W, Sun W, et al. Peripherally inserted central catheter-related complications in cancer patients: a prospective study of over 50,000 catheter days. J Vasc Access 2017; 21;18:153-7.

6. Şirinoğlu M, Soysal A, Karaaslan A, et al. The diagnostic value of soluble urokinase plasminogen activator receptor (suPAR) compared to C-reactive protein (CRP) and procalcitonin (PCT) in children with systemic 
inflammatory response syndrome (SIRS). J Infect

Chemother 2017;23:17-22.

7. Plesko M, Suvada J, Makohusova M, et al. The role of CRP, PCT, IL-6 and presepsin in early diagnosis of bacterial infectious complications in paediatric haematooncological patients. Neoplasma 2016;63:752-60.

8. Shilpakar R, Paudel BD, Neupane P, et al. Procalcitonin and C-Reactive Protein As Markers of Bacteremia in Patients With Febrile Neutropenia Who Receive Chemotherapy for Acute Leukemia: A Prospective Study From Nepal. J Glob Oncol 2019;5:1-6.

9. Stankovic Stojanovic K, Hentgen V, Fellahi S, et al. Concordance between CRP and SAA in familial Mediterranean fever during attack-free period: A study of 218 patients. Clin Biochem 2017;50:206-9.

10. Bishnoi NK, Singh N, Sharma R. A prospective observational study to evaluate serum Procalcitonin as a bio marker of sepsis in critically ill patients and it's correlation with their clinicoetiological profi le. J Assoc

Cite this article as: Chen J, Huang J, Wang T, Xie C. Analysis of the relationship between serum amyloid protein A, procalcitonin, C-reactive protein, and peripherally inserted central catheter infection in patients with malignant tumor. Ann Palliat Med 2021;10(5):5359-5365. doi: 10.21037/apm-21-796
Physicians India 2020;68:95.

11. Viswateja K, I U, Dayanand. Role of Procalcitonin and Other Biomarkers in Predicting Sepsis in CKD Patients on Haemodialysis. J Assoc Physicians India 2020;68:77.

12. Levy S, Rottenstreich A, Kalish Y. Risk factors associated with the development of upper extremity deep vein thrombosis following peripherally inserted central catheter insertion. Harefuah 2019;158:150-4.

13. Digre A, Nan J, Frank M, et al. Heparin interactions with apoA1 and SAA in inflammation-associated HDL. Biochem Biophys Res Commun 2016;474:309-14.

14. Niu T, Liu Y, Zhu F, et al. Time-resolved fluorescent immunoassay-based combined detection of procalcitonin, C-reactive protein, heparin binding protein, and serum amyloid A1 to improve the diagnostic accuracy of early infection. J Clin Lab Anal 2019;33:e22694.

(English Language Editor: J. Gray) 